\title{
Practice of Clinical Teaching Reform based on Health Belief Model
}

\author{
Fenggang Pan \\ Yichun Vocational Technology College, Yichun 336000, China \\ nior_0417@sina.com
}

\begin{abstract}
The purpose of this study is to study the effect of this health education mode on the practice of clinical teaching reform based on the health belief model. We made an attempt and satisfaction survey on three teaching methods of clinical medicine courses for 90 students in our school. The results show that the clinical teaching method based on the health belief model is novel, clear in thinking, focused and easy to understand and remember. At the same time, taking cases to analyze, it strengthens the training of clinical thinking, cultivates a kind of ability and stimulates learning interest. This shows that the practice of clinical teaching reform based on health belief model is basically complete and feasible, and provides a set of scientific and reasonable methods for clinical teaching in the future.
\end{abstract}

Keywords: Health Belief Model; Clinical Teaching; Reform.

\section{Introduction}

Clinical teaching is different from basic teaching, which is embodied in the fact that clinical teachers have three tasks: medical treatment, teaching and scientific research. Because of the hospital's own management system, the actual situation of medical work, the relatively weak awareness of teaching, poor teaching conditions and many other factors, it has restricted the scale and development of medical clinical teaching and directly affected the steady improvement of clinical teaching quality [1]. In order to keep nursing education systematic and practical, clinical nursing teaching undertakes the important task of imparting clinical nursing professional knowledge and transforming knowledge into ability training.

As an important part of medical and health service industry, hospitals play an important role in health education. Health education is a kind of health education activity, which is a planned and purposeful activity for patients or healthy people, formulated by nurses. It has nursing characteristics and plays an important role in the physiological, psychological, cultural, spiritual and social adaptability of patients and their families [2-3].

\section{Overview of Health Belief Model}

Health belief model is a theory to study the relationship between people's health belief and health behavior, which was first put forward by Hockbaum in 1958, and then revised and perfected by social psychologist Beck Becker and other psychologists. It refers to a theoretical model to explain and predict individual's health-related behavior, and to guide health education and behavior intervention by using psychological methods, combining the viewpoints of need motivation theory, cognitive theory and value expectation theory.

This theory mainly consists of the following seven aspects (Figure 1), namely:

(1) Perceived susceptibility to diseases: It refers to people's cognition of the occurrence degree of certain diseases.

(2) Perceived severity of the disease: It refers to people's cognition of the degree of harm caused by a certain disease, including clinical prognosis and social consequences.

(3) Perceived behavioral benefits: that is, whether people follow or give up certain behaviors will have an impact on alleviating the disease or reducing the risk of its occurrence.

(4) Perceived behavior disorder: It refers to the cognition of the difficulty of taking or abandoning a certain behavior. 
Volume 14 (2021)

(5) Self-efficacy: refers to the self-confidence of implementing or giving up a certain behavior, which is a person's judgment of his own behavior ability.

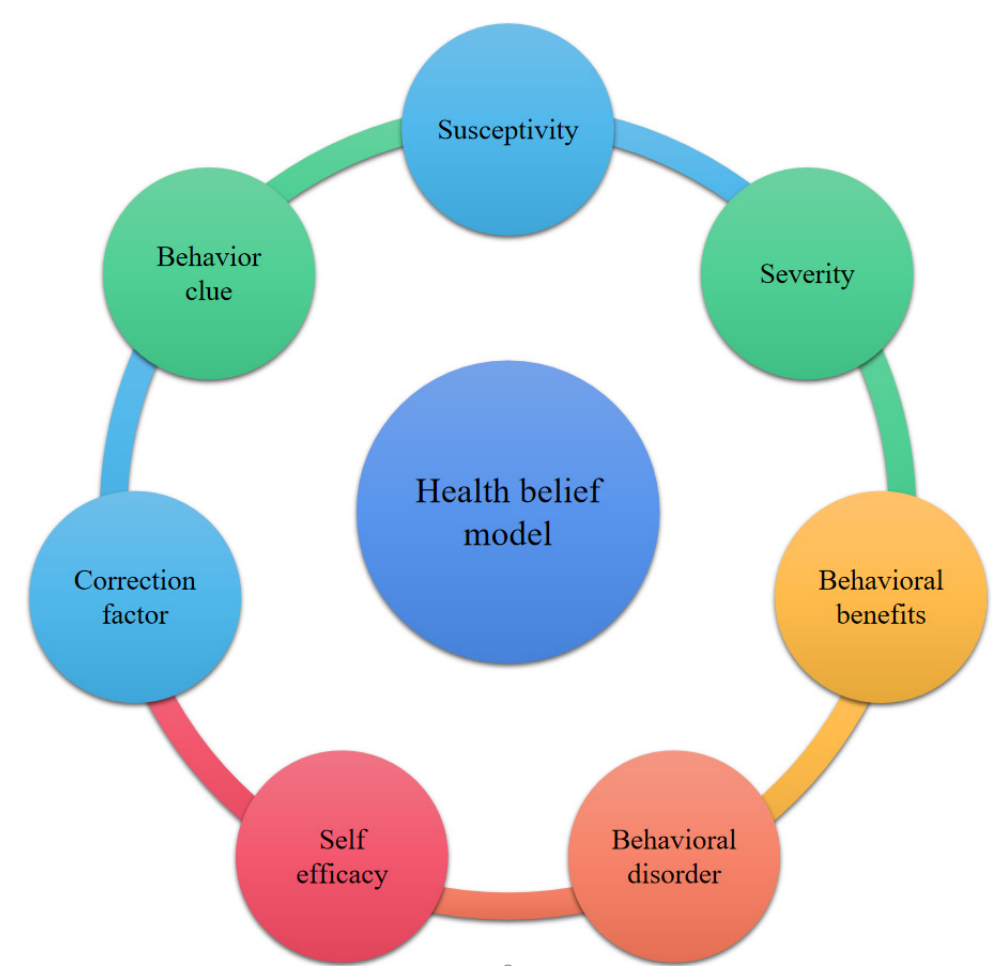

Figure 1. Health belief model

(6) Modifier: It mainly includes some characteristics related to individuals, such as gender, age, race, personality traits, social class, peer influence and knowledge of disease and health.

(7) Behavioral cues: such as the mass media's propaganda of disease knowledge, the health behaviors advocated by medical workers, and the previous medical history of family members or friends, etc., can be used as behavioral cues to influence individuals to take healthy behaviors [7].

Self-efficacy, correction factors and behavioral cues have different influences on susceptibility cognition, severity cognition, behavioral benefit cognition and behavioral disorder cognition in the health belief model, thus affecting behavioral changes.

\section{Disadvantages of Traditional Clinical Teaching Methods}

Small lecture is an important part of clinical nursing teaching, which is a teaching integrating basic theoretical knowledge and practical clinical professional knowledge. The content of preparing lessons for clinical teachers from the theory of diseases to the application of clinical nursing knowledge is rich and detailed. However, the teaching methods are still inseparable from the traditional mode, and teaching is the main task, with students taking notes from beginning to end and always being passively accepted.

Because the basic theoretical knowledge has been studied repeatedly in school and the examination has been strengthened, students cannot arouse enough interest and attention to the re-study at the known level, and they are disgusted with boredom, wasting time, lacking learning initiative and enthusiasm, and the quality and effect of small lectures cannot reach the desired goal.

\section{Method}

In the practice of clinical theory teaching reform, we have tried a variety of new teaching methods. In order to explore the teaching effect of different teaching methods and the adaptability of this 
method to students, we made the following three attempts and satisfaction surveys on teaching methods of clinical medicine courses for 90 students in school.

\subsection{Traditional Forms of Teaching}

Taking a certain disease as the longitudinal axis, it is carried out according to the current teaching material system, that is, it follows a closely arranged and formalized clue of "logic-deduction". For example, in the chapter of lung cancer, it is taught in the order of epidemiology, etiology, pathogenesis, pathology, clinical manifestation, auxiliary examination, differential diagnosis and treatment. Teaching is basically taught by one teacher, and there is almost no communication and interaction between teachers and students.

\subsection{Multimedia Courseware Teaching}

The multimedia courseware made by PPT is divided into two parts: on-demand projection with clear navigation and automatic projection with explanation. For example, asthma course adopts the former. It is characterized by a large number of links to the latest relevant information for counseling, and a large number of exercises and correct answers for practice and self-test. Equipped with English vocabulary for bilingual teaching. Teaching is also conducted by one teacher, without communication between teachers and students.

\subsection{Clinical Teaching based on Health Belief Model}

To enable the nursing clients to achieve the purpose of changing behaviors. By applying the health belief model, nurses can evaluate the individual's health belief and the factors affecting their health belief, and take effective measures to establish and enhance the health belief of clients, so that they can voluntarily adopt healthy behaviors, thus achieving the purpose of promoting health.

Evaluating the individual's awareness of the severity of the disease: including evaluating the individual's awareness of the clinical consequences caused by the disease and the social consequences caused by the disease. The psychological effects are mainly anger, guilt, anxiety, fear, sadness, disappointment, depression and so on. Assess the individual's awareness of the threat of a certain disease or risk factors: according to the epidemic trend, susceptible factors, risk factors and susceptible population of a specific disease, assess the individual's awareness of the susceptibility to the disease.

\section{Result}

See Figure 2 below for the survey results of three classroom teaching methods and their effects.



Figure 2. Investigation results of three classroom teaching methods and their effects 


\subsection{Satisfaction with Traditional Teaching Forms}

Most students report that the course is rich in content, reasonable in arrangement, focused, clear in organization and easy to remember, but lacks in thinking training. A few students think the content is too much and boring.

\subsection{Satisfaction of Multimedia Courseware Teaching}

Most students think that this method is novel, detailed in content, strong in sensory stimulation, illustrated and vivid in image. A small number of students think that the courseware is too miscellaneous, boring, difficult to review, fast and too late to record. Although the results of single survey are better than the traditional teaching methods, the comprehensive satisfaction decreases because of several problems of projection instruments.

\subsection{Satisfaction of Clinical Teaching based on Health Belief Model}

Students agree that this method is novel, clear-cut, focused, and easy to understand and remember. At the same time, it takes cases to analyze, which strengthens clinical thinking training, cultivates a kind of ability, and stimulates learning interest.

\section{Discussion}

To sum up, it is not difficult to see that, in fact, the above-mentioned different teaching methods have their own characteristics and advantages and disadvantages. For example, the traditional teaching form is clear and systematic, which is conducive to the arrangement, arrangement and optimization of knowledge and lays a deep theoretical foundation for students. However, it is difficult to achieve the purpose of clinical thinking training, and it is easy to ignore the development of students' personality and the formation of thinking for seeking differences, which restricts their initiative and creative potential.

\subsection{Effect of Teaching Reform based on Health Belief Model}

(1) Improve students' interest in learning and thirst for knowledge

The teaching method based on the health belief model is to guide students to apply knowledge and acquire new knowledge through independent thinking according to the existing basic knowledge. From its psychological mechanism, it is exploratory and can stimulate students' thinking activities. The personal attempt of teaching role transformation, the tension and novelty from passive acceptance to active participation, has greatly improved students' interest in learning and thirst for knowledge.

(2) Train students' ability to collect and use information

In order to participate in the discussion, students put forward their own opinions, and in order to output more knowledge in teaching, lecturers cultivate the knowledge of collecting newspaper clippings and extracurricular books consciously, to increase new knowledge, expand knowledge capacity and show their professional level. Students understand the value of information, have the ability to use information, have a strong interest in collecting information, and exercise students' ability of social interaction.

(3) Cultivate students' clinical thinking

The case education based on the health belief model is often diversified except the simulation. In teaching, the only correct answer is not deliberately sought, and the training of thinking methods is emphasized. At the same time, it is more enlightening, which enables students to think and explore practical problems and enliven their thinking. Therefore, through discussion and active thinking, students' analytical ability and problem-solving ability have been greatly improved, and the teaching method designed with ability training as the main line has promoted the transformation from knowledge to ability. 


\subsection{Teaching Inspiration}

In the clinical teaching practice, the author thinks that the following points are helpful to improve the teaching effect:

(1) Let individuals perceive the benefits and obstacles of healthy behavior

It is not enough for individuals to realize the harmfulness and severity of diseases. It is important to realize the benefits and obstacles of healthy behaviors. Nurses should make individuals realize that giving up behaviors harmful to health can really get the preventive effect, that is, the effectiveness of behaviors. People's awareness of the difficulties in promoting health behavior is the prerequisite for the behavior to be consolidated and sustained. Perception of susceptibility and severity really provides energy and strength for action; However, only when the public is aware of the benefits and can understand all the difficulties before they are determined to overcome them, can he really find the way of behavior.

(2) Strengthen the influence of restrictive factors on individuals' health behaviors

Specific measures are as follows: First, provide individuals with relevant newspapers and magazines, health brochures, health brochures, relevant TV program channels and broadcast time, etc., so that individuals can obtain relevant health education knowledge from multiple channels in time; Second, if an individual's relatives or friends have the experience of related diseases, they can help the individual understand the importance of health and establish a correct belief in health. Third, introduce relevant health behaviors to relatives and friends of individuals, and use their positive comments on health behaviors to promote individuals to adopt healthy behaviors; Fourth, health behavior education should be carried out in the ward, so that patients can communicate with each other and obtain health information indirectly.

(3) Imitation case teaching

Cases presented in front of students are actual cases with real content and flesh. Students can make analysis and judgment from the actual situation, instead of making unrealistic dreams, and cultivate students' ability to solve various problems in practice, so that teaching not only pays attention to knowledge, but also pays more attention to the ability of those who master knowledge.

\section{Summary}

Using the health belief model in health education, nurses can choose the education content and plan according to the level of patients' health belief, so that nurses can carry out targeted individual nursing, and individuals have a correct and full understanding of their own behaviors and diseases, thus changing bad behaviors, consciously adopting healthy behaviors and improving their health level. Through the satisfaction analysis of clinical teaching based on the health belief model, it is preliminarily confirmed that the teaching method is basically complete and feasible, which provides reference for future clinical teaching.

\section{References}

[1] Kuloko Shen, Wang Taotao, Gao Min, et al. Evaluation of intervention effect of health education based on health belief model on hypertension patients in community [J]. Chinese Journal of Preventive Medicine, 2020, 54(02):155-159.

[2] Zhong Huimei, Jiang Hongmei, Rei Dan. Clinical effect of perinatal education based on health belief model on preventing postpartum depression [J]. Forum of Primary Medical Sciences, 2020, v.24; No. 620 (32): 7-9.

[3] Zhang Xiaoxia. Application of health education based on health belief model in oral basic treatment for outpatients with periodontal disease complicated with diabetes mellitus [J]. Chinese Community Physician, 2019, v.35; No.798(36):186-187.

[4] Lv Guizhi. the influence of path-based health education based on health belief model on patients with chronic hepatitis b [J]. nursing practice and research, 2019, v.16(05):41-43. 
[5] Li Peilin. Evaluation of the application effect of health belief model in health education for patients with chronic atrophic gastritis [J]. Electronic Journal of Practical Clinical Nursing, 2019, v.4(33):185-185.

[6] Shi Zhongli, Li Yinping, Feng Yutao, et al. Influence of health education based on health belief model on medication belief and medication compliance of elderly patients with depression [J]. Contemporary Nurses (Specialist Edition), 2019, 026(012):157-159.

[7] Yan LAN, Lu quan-Zhen, Lu ren-yi, et al. exploration and practice of clinical pharmacotherapy teaching reform in pharmacy specialty [J]. pharmaceutical service and research, 2020, v.20(01):80-82. 